Article

\title{
On Two Open Problems on Double Vertex-Edge Domination in Graphs
}

\author{
Fang Miao ${ }^{1,2}$, Wenjie Fan 1,2, Mustapha Chellali ${ }^{3}$, Rana Khoeilar ${ }^{4}$, \\ Seyed Mahmoud Sheikholeslami ${ }^{4, *}$ and Marzieh Soroudi ${ }^{4}$ \\ 1 The Big Data Research Institute, Chengdu University, Chengdu 610106, China; miaofang@126.com (F.M.); \\ fanwenjie@cdu.edu.cn (W.F.) \\ 2 Key Laboratory of Pattern Recognition and Intelligent Information Processing, Chengdu 610106, China \\ 3 LAMDA-RO Laboratory, Department of Mathematics, University of Blida, B.P. 270 Blida, Algeria; \\ m_chellali@yahoo.com \\ 4 Department of Mathematics, Azarbaijan Shahid Madani University, Tabriz 51368, Iran; \\ khoeilar@azaruniv.ac.ir (R.K.); m.soroudi@azaruniv.ac.ir (M.S.) \\ * Correspondence: s.m.sheikholeslami@azaruniv.ac.ir
}

Received: 13 September 2019; Accepted: 21 October 2019; Published: 24 October 2019

\begin{abstract}
A vertex $v$ of a graph $G=(V, E)$, ve-dominates every edge incident to $v$, as well as every edge adjacent to these incident edges. A set $S \subseteq V$ is a double vertex-edge dominating set if every edge of $E$ is ve-dominated by at least two vertices of $S$. The double vertex-edge domination number $\gamma_{d v e}(G)$ is the minimum cardinality of a double vertex-edge dominating set in $G$. A subset $S \subseteq V$ is a total dominating set (respectively, a 2-dominating set) if every vertex in $V$ has a neighbor in $S$ (respectively, every vertex in $V-S$ has at least two neighbors in $S$ ). The total domination number $\gamma_{t}(G)$ is the minimum cardinality of a total dominating set of $G$, and the 2-domination number $\gamma_{2}(G)$ is the minimum cardinality of a 2-dominating set of G. Krishnakumari et al. (2017) showed that for every triangle-free graph $G, \gamma_{d v e}(G) \leq \gamma_{2}(G)$, and in addition, if $G$ has no isolated vertices, then $\gamma_{d v e}(G) \leq \gamma_{t}(G)$. Moreover, they posed the problem of characterizing those graphs attaining the equality in the previous bounds. In this paper, we characterize all trees $T$ with $\gamma_{d v e}(T)=\gamma_{t}(T)$ or $\gamma_{d v e}(T)=\gamma_{2}(T)$.
\end{abstract}

Keywords: vertex-edge dominating set; double vertex-edge dominating set; total dominating set; 2-dominating set

\section{Introduction}

In this paper, $G$ is a simple nontrivial connected graph with vertex set $V=V(G)$ and edge set $E=E(G)$. The order $|V|$ of $G$ is denoted by $n=n(G)$. For a vertex $v \in V$, the "open neighborhood" of $v$ is the set $N(v)=\{u \in V(G): u v \in E(G)\}$ and the "closed neighborhood" of $v$ is the set $N[v]=N(v) \cup\{v\}$. The "degree" of a vertex $v \in V$ is $\operatorname{deg}_{G}(v)=|N(v)|$. A vertex of degree one is called a "pendant vertex" or a "leaf" and its neighbor is called a "support vertex". A "strong support vertex" is a support vertex adjacent to at least two leaves and an "end support vertex" is a support vertex having at most one non-leaf neighbor. If $v$ is a support vertex, then $L_{v}$ denotes the set of leaves adjacent to $v$. A "pendant path" $P$ of a graph $G$ is an induced path starting from a leaf and containing only vertices of degree two in $G$ as inner vertices. The "distance" between two vertices $u$ and $v$ in a connected graph $G$ is the length of a shortest $u v$-path in $G$. The "diameter" of connected graph $G$, denoted by $\operatorname{diam}(G)$, is the maximum value among minimum distances between all pairs of vertices of $G$. For a vertex $v$ in a rooted tree $T$, let $C(v)$ and $D(v)$ denote the set of children and descendants of $v$, respectively, and let $D[v]=D(v) \cup\{v\}$. Also, the "depth" of $v$, depth $(v)$, is the largest distance 
from $v$ to a vertex in $D(v)$. The "maximal subtree" at $v$ is the subtree of $T$ induced by $D[v]$, and is denoted by $T_{v}$. We write $P_{n}$ for a path of order $n$. A "double star" $D S_{p, q}$ is a tree containing exactly two non-pendant vertices, one of which is adjacent to $p$ leaves and the other is adjacent to $q$ leaves. If $A \subseteq V(G)$ and $f$ is a mapping from $V(G)$ into some set of numbers, then $f(A)=\sum_{x \in A} f(x)$, and the sum $f(V(G))$ is called the "weight" $\omega(f)$ of $f$.

A subset $S \subseteq V$ is a "2-dominating set", abbreviated 2d-set, of $G$ if every vertex in $V \backslash S$ is adjacent to at least two vertices in $S$ and it is a "total dominating set" (td-set), if every vertex in $V$ is adjacent to a vertex in $S$. The "2-domination number", $\gamma_{2}(G)$ (respectively, "total domination number", $\gamma_{t}(G)$ ) of $G$, is the minimum cardinality of a 2d-set (respectively, td-set) of $G$. A 2d-set (respectively, td-set) of $G$ with minimum cardinality is called a $\gamma_{2}(G)$-set (respectively, $\gamma_{t}(G)$-set). The literature on the subject of domination and total domination in graphs has been surveyed and detailed in two books [1,2].

A vertex $v$ is said to "ve-dominate" every edge incident to any vertex in $N[v]$. A set $S \subseteq V$ is a "vertex-edge dominating set" (or simply, a ve-dominating set) if for every edge $e \in E$, there exists a vertex $v \in S$ that ve-dominates $e$. The minimum cardinality of a ve-dominating set of $G, \gamma_{v e}(G)$, is called the "vertex-edge domination number". Vertex-edge domination was introduced by Peters [3] in his $1986 \mathrm{PhD}$ thesis and studied further in [4-10].

A vertex-edge dominating set $D \subseteq V$ is called a "double vertex-edge dominating set (or simply, a dve-dominating set) of $G$, if every edge of $E$ is ve-dominated by at least two vertices of $D$. The "double vertex-edge domination number" of $G, \gamma_{d v e}(G)$, is the minimum cardinality of a double ve-dominating set. The concept of double vertex-edge domination in graphs was introduced by Krishnakumari, Chellali, and Venkatakrishnan in [11], where they proved the following results.

Proposition 1 ([11]). For every triangle-free graph $G$ without isolated vertices,

$$
\gamma_{d v e}(G) \leq \gamma_{t}(G)
$$

Proposition 2 ([11]). For every graph $G$,

$$
\gamma_{d v e}(G) \leq \gamma_{2}(G)
$$

Moreover, the authors [11] posed these two problems.

Problem 1. Characterize all connected graphs $G$ with $\gamma_{d v e}(G)=\gamma_{2}(G)$.

Problem 2. Characterize all nontrivial trees $T$ with $\gamma_{d v e}(T)=\gamma_{t}(T)$.

In this paper, we settle the above open problems for trees by providing a constructive characterization of all trees $T$ with $\gamma_{d v e}(T)=\gamma_{2}(T)$ or $\gamma_{d v e}(T)=\gamma_{t}(T)$.

\section{Preliminaries}

In this section, we provide some definitions and observations that will be useful throughout the paper. We introduce some sets of vertices satisfying special condition.

Definition 1. Let $u$ be a vertex of a graph $G$. A subset $S$ of vertices is said to be an almost dve-dominating set with respect to $u$ if the following conditions are fulfilled: (i) any edge not incident to $u$, is ve-dominated by at least two vertices in $S$, and (ii) any edge incident to $u$ is ve-dominated by at least one vertex in $S$. Define

$$
\gamma_{\text {dve }}(G ; u)=\min \{|S|: S \text { is an almost dve-dominating set with respect to } u\} \text {. }
$$

Clearly, any dve-dominating set on $G$ is an almost dve-dominating set with respect to any vertex of $G$ and so $\gamma_{d v e}(G ; u) \leq \gamma_{d v e}(G)$ for each $u \in V(G)$. Define

$$
W_{G}^{1}=\left\{u \in V(G) \mid \gamma_{d v e}(G ; u)=\gamma_{d v e}(G)\right\} .
$$


Definition 2. For a graph $\mathrm{G}$, define

$$
\begin{aligned}
& W_{G}^{2}=\left\{v \in V(G) \mid \text { there exists a } \gamma_{\text {dve }}(G) \text {-set } S \text { such that }\left|S \cap N_{G}[v]\right| \geq 1\right\}, \\
& W_{G}^{3}=\left\{v \in V(G) \mid \text { there exists a } \gamma_{2}(G) \text {-set } S \text { such that } v \in S\right\}, \\
& W_{G}^{4}=\{v \in V(G) \mid \text { there exists no dve-dominating set } S \text { of } G \text { such that } v \in S\} .
\end{aligned}
$$

Observation 1. All leaves of a graph $G$ are in every 2-dominating set of $G$.

Observation 2. For any connected graph $G$ with diameter at least three, there exists a $\gamma_{t}(G)$-set (respectively, $\gamma_{\text {dve }}(G)$-set) that contains no leaf of $G$.

Proof. Among all $\gamma_{t}(G)$-sets, let $D$ be one containing the minimum number of leaves of $G$. Assume that there exists a leaf of $G$, say $x$ with $x \in D$. Let $y$ be the support vertex adjacent to $x$. Clearly, $y \in D$ (else $x$ will be without neighbors in $D$ ), and $A=N(y)-L_{v} \neq \varnothing$ (as $G$ has diameter at least three). If $A \cap D \neq \varnothing$, then $D-\{x\}$ is a td-set of $G$, a contradiction. Therefore, $A \cap D=\varnothing$. Then, replacing $x$ in $D$ by any vertex of $A$, provides a $\gamma_{t}(G)$-set containing fewer leaves than $D$, contradicting our choice of $D$.

A similar argument can be used to prove also that $G$ has a $\gamma_{d v e}(G)$-set that contains no leaf.

Proposition 3. Let $G$ be a nontrivial connected graph and $u \in V(G)$ a vertex of degree at least two which is a support vertex or adjacent to an end-support vertex. If $G^{\prime}$ is the graph obtained from $G$ by adding a new vertex $v$ connected by an edge to $u$, then $\gamma_{d v e}(G)=\gamma_{d v e}\left(G^{\prime}\right)$ and $\gamma_{t}(G)=\gamma_{t}\left(G^{\prime}\right)$.

Proof. If $G^{\prime}$ is a star, then the results are immediate. Suppose $G^{\prime}$ is not a star. Thus, both $G^{\prime}$ and $G$ have diameter at least three. By Observation 2, any $\gamma_{t}(G)$-set (respectively, $\gamma_{t}\left(G^{\prime}\right)$-set) containing no leaves contains $u$, and so is a td-set of $G^{\prime}$ (respectively, $G$ ) yielding $\gamma_{t}\left(G^{\prime}\right) \leq \gamma_{t}(G)$ (respectively, $\left.\gamma_{t}(G) \leq \gamma_{t}\left(G^{\prime}\right)\right)$. Therefore, $\gamma_{t}\left(G^{\prime}\right)=\gamma_{t}(G)$.

Assume now that $D$ is a $\gamma_{d v e}\left(G^{\prime}\right)$-set containing no leaves. Then to ve-dominate the edge $u v$, we may assume that $D \cap(N[u]-\{v\}) \neq \varnothing$ and $D$ is clearly a dve-dominating set of $G$ implying that $\gamma_{d v e}\left(G^{\prime}\right) \geq \gamma_{d v e}(G)$. On the other hand, clearly any $\gamma_{d v e}(G)$-set containing no leaves is a double ve-dominating set of $G^{\prime}$ and so $\gamma_{d v e}\left(G^{\prime}\right) \leq \gamma_{d v e}(G)$. Thus, $\gamma_{d v e}\left(G^{\prime}\right)=\gamma_{d v e}(G)$.

Proposition 4. Let $G$ be a nontrivial connected graph and $u \in V(G)$ such that $u x_{1} x_{2}$ is a path in $G$ in which $\operatorname{deg}_{G}\left(x_{1}\right)=2$ and $\operatorname{deg}_{G}\left(x_{2}\right)=1$. If $G^{\prime}$ is a graph obtained from $G$ by attaching a path $P_{2}=y_{1} y_{2}$ and joining $y_{1}$ to $\mathrm{u}$, then $\gamma_{t}\left(G^{\prime}\right)=\gamma_{t}(G)+1, \gamma_{d v e}\left(G^{\prime}\right)=\gamma_{d v e}(G)+1$ and $\gamma_{2}\left(G^{\prime}\right)=\gamma_{2}(G)+1$.

Proof. Clearly, any $\gamma_{t}(G)$-set (respectively, $\gamma_{t}\left(G^{\prime}\right)$-set) containing no leaves, contains $u, x_{1}$ (respectively, $\left.u, x_{1}, y_{1}\right)$ and for which adding (respectively, removing) vertex $y_{1}$ yields a td-set of $G^{\prime}$ (respectively, $G)$. Therefore, $\gamma_{t}\left(G^{\prime}\right) \leq \gamma_{t}(G)+1$ (respectively, $\left.\gamma_{t}(G) \leq \gamma_{t}\left(G^{\prime}\right)-1\right)$ ), and so $\gamma_{t}\left(G^{\prime}\right)=\gamma_{t}(G)+1$. Similarly, we can see that $\gamma_{\text {dve }}\left(G^{\prime}\right)=\gamma_{\text {dve }}(G)+1$ and $\gamma_{2}\left(G^{\prime}\right)=\gamma_{2}(G)+1$.

Proposition 5. Let $G$ be a nontrivial connected graph and let $u$ be a vertex of $G$. If $G^{\prime}$ is the graph obtained from $G$ by adding a path $P_{4}=x_{1} x_{2} x_{3} x_{4}$ and joining $u$ to $x_{1}$, then $\gamma_{t}\left(G^{\prime}\right)=\gamma_{t}(G)+2$ and $\gamma_{d v e}\left(G^{\prime}\right) \geq \gamma_{d v e}(G)+2$. Moreover, if $u \in W_{G^{\prime}}^{2}$ then $\gamma_{d v e}\left(G^{\prime}\right)=\gamma_{d v e}(G)+2$.

Proof. Clearly, any $\gamma_{t}(G)$-set can be extended to a td-set of $G^{\prime}$ by adding $x_{2}, x_{3}$, and so $\gamma_{t}\left(G^{\prime}\right) \leq$ $\gamma_{t}(G)+2$

To prove the inverse inequality, let $v \in N_{G}(u)$ and let $D$ be a $\gamma_{t}\left(G^{\prime}\right)$-set containing no leaves. Then, $x_{2}, x_{3} \in D$. If $v \in D$ or $x_{1} \notin D$, then $D \backslash\left\{x_{2}, x_{3}\right\}$ is a td-set of $G$ yielding $\gamma_{t}\left(G^{\prime}\right) \geq \gamma_{t}(G)+2$. Thus, assume that $v \notin D$ and $x_{1} \in D$. Then, $\left(D \backslash\left\{x_{1}, x_{2}, x_{3}\right\}\right) \cup\{v\}$ is a total dominating set of $G$ yielding $\gamma_{t}\left(G^{\prime}\right) \geq \gamma_{t}(G)+2$. Therefore, $\gamma_{t}\left(G^{\prime}\right)=\gamma_{t}(G)+2$. 
Similarly, we can see that $\gamma_{d v e}\left(G^{\prime}\right) \geq \gamma_{d v e}(G)+2$. Now, if $u \in W_{G^{\prime}}^{2}$, then clearly some $\gamma_{d v e}(G)$-set contains a vertex $N_{G}[u]$, and so it can be extended to a dve-dominating set of $G^{\prime}$ by adding $x_{2}, x_{3}$ and thus $\gamma_{d v e}\left(G^{\prime}\right) \leq \gamma_{d v e}(G)+2$. Therefore, $\gamma_{d v e}\left(G^{\prime}\right)=\gamma_{d v e}(G)+2$ when $u \in W_{G}^{2}$.

Proposition 6. Let $G$ be a nontrivial connected graph and let $u$ be a vertex of $G$, such that $u x_{1} x_{2}$ is a pendant path in $G$ with $\operatorname{deg}_{G}\left(x_{2}\right)=1$. If $G^{\prime}$ is the graph obtained from $G$ by adding a path $P_{3}=y_{1} y_{2} y_{3}$ and joining $u$ to $y_{1}$, then $\gamma_{t}\left(G^{\prime}\right)=\gamma_{t}(G)+2, \gamma_{2}\left(G^{\prime}\right)=\gamma_{2}(G)+2$ and $\gamma_{d v e}\left(G^{\prime}\right)=\gamma_{d v e}(G)+2$.

Proof. Clearly, any $\gamma_{t}(G)$-set (respectively, $\gamma_{t}\left(G^{\prime}\right)$-set) $D$ containing no leaves contains $u, x_{1}$ (respectively, $u, x_{1}, y_{1}, y_{2}$ ) and so $D \cup\left\{y_{1}, y_{2}\right\}$ (respectively, $D-\left\{y_{1}, y_{2}\right\}$ ) is a td-set of $G^{\prime}$ (respectively, $G)$ yielding $\gamma_{t}\left(G^{\prime}\right)=\gamma_{t}(G)+2$. Similarly, we can easily see that $\gamma_{2}\left(G^{\prime}\right)=\gamma_{2}(G)+2$.

Assume now that $D$ is a $\gamma_{d v e}\left(G^{\prime}\right)$-set containing no leaves. Obviously, to dve-dominate the edges $x_{1} x_{2}, y_{2} y_{3}$, we must have $u, x_{1}, y_{1}, y_{2} \in D$ and thus $D-\left\{y_{1}, y_{2}\right\}$ is a dve-dominating of $G$. Thus, $\gamma_{d v e}\left(G^{\prime}\right) \geq \gamma_{d v e}(G)+2$. The equality is obtained from the fact that any $\gamma_{d v e}(G)$-set can be extended to a dve-dominating set of $G^{\prime}$ by adding $y_{1}, y_{2}$.

Proposition 7. Let $G$ be a nontrivial connected graph and let $u$ be a vertex of $G$ such that $u x_{1} x_{2} x_{3}$ is a pendant path in $G$ with $\operatorname{deg}_{G}\left(x_{3}\right)=1$. If $G^{\prime}$ is the graph obtained from $G$ by adding a path $P_{3}=y_{1} y_{2} y_{3}$ and joining $u$ to $y_{1}$, then $\gamma_{t}\left(G^{\prime}\right)=\gamma_{t}(G)+2$ and $\gamma_{d v e}\left(G^{\prime}\right) \leq \gamma_{d v e}(G)+2$. Furthermore, if $u \in W_{G^{\prime}}^{1}$, then $\gamma_{d v e}\left(G^{\prime}\right)=\gamma_{d v e}(G)+2$.

Proof. As in the proof of Proposition 6, we can see that $\gamma_{t}\left(G^{\prime}\right)=\gamma_{t}(G)+2$. The inequality $\gamma_{d v e}\left(G^{\prime}\right) \leq$ $\gamma_{d v e}(G)+2$ follows from the fact that any $\gamma_{d v e}(G)$-set can be extended to a dve-dominating set of $G^{\prime}$ by adding $y_{1}, y_{2}$. Assume now that $u \in W_{G}^{1}$ and let $D$ be a $\gamma_{d v e}\left(G^{\prime}\right)$-set containing no leaves. Then we must have $x_{1}, x_{2}, y_{1}, y_{2} \in D$, and thus $D-\left\{y_{1}, y_{2}\right\}$ is an almost dve-dominating set of $G$ with respect to $u$. We deduce from $u \in W_{G}^{1}$ that $\left|D-\left\{y_{1}, y_{2}\right\}\right| \geq \gamma_{d v e}(G ; u)=\gamma_{d v e}(G)$ and so $\gamma_{d v e}\left(G^{\prime}\right) \geq \gamma_{d v e}(G)+2$. Hence $\gamma_{d v e}\left(G^{\prime}\right)=\gamma_{\text {dve }}(G)+2$ when $u \in W_{G}^{1}$.

Let $k \geq 2$ be an integer and $H_{k}$ be the graph obtained from the complete bipartite graph $K_{1, k}$ (also known by a star) by subdividing every edge twice (see Figure 1).

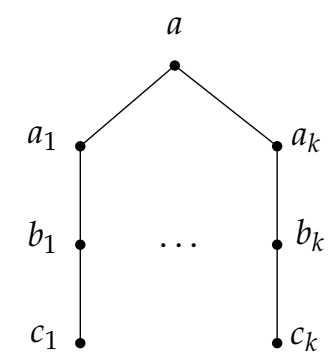

Figure 1. The graph $H_{k}(k \geq 2)$ which is defined above.

Proposition 8. Let $G$ be a nontrivial connected graph and let $u$ be a vertex of $G$. If $G^{\prime}$ is the graph obtained from $G$ by adding the graph $H_{k}$ and joining $u$ to the center vertex of $H_{k}$. Then, $\gamma_{2}\left(G^{\prime}\right)=\gamma_{2}(G)+2 k$ and $\gamma_{d v e}\left(G^{\prime}\right)=\gamma_{d v e}(G)+2 k$.

Proof. Clearly, any $\gamma_{2}(G)$-set can be extended to a $2 \mathrm{~d}$-set of $G^{\prime}$ by adding $a_{i}, c_{i}$ for $1 \leq i \leq k$, implying that $\gamma_{2}\left(G^{\prime}\right) \leq \gamma_{2}(G)+2 k$.

To prove the inverse inequality, let $D$ be $\gamma_{2}\left(G^{\prime}\right)$-set. Obviously, $c_{i} \in D$ for each $i$. Now to 2-dominate the vertex $b_{i}$ we must have $\left|D \cap\left\{a_{i}, b_{i}\right\}\right| \geq 1$ for each $i$. If $a \notin D$, then $D-V\left(H_{k}\right)$ is a 2d-set of $G$ and if $a \in D$, then $\left(D-V\left(H_{k}\right)\right) \cup\{u\}$ is a 2d-set of $G$. In either case, $\gamma_{2}\left(G^{\prime}\right) \geq \gamma_{2}(G)+2 k$, and thus $\gamma_{2}\left(G^{\prime}\right)=\gamma_{2}(G)+2 k$. Similarly, we can easily see that $\gamma_{d v e}\left(G^{\prime}\right)=\gamma_{d v e}(G)+2 k$. 
Observation 3. Let $G$ be a triangle-free graph without isolated vertices, and let $H$ be a subgraph of $G$.

1. If $\gamma_{d v e}(H)=\gamma_{t}(H), \gamma_{t}(G) \leq \gamma_{t}(H)+s$ and $\gamma_{d v e}(G) \geq \gamma_{d v e}(H)+s$ for some non-negative integer $s$, then $\gamma_{d v e}(G)=\gamma_{t}(G)$.

2. If $\gamma_{d v e}(G)=\gamma_{t}(G), \gamma_{d v e}(G) \leq \gamma_{d v e}(H)+s$ and $\gamma_{t}(G) \geq \gamma_{t}(H)+s$ for some non-negative integer $s$, then $\gamma_{d v e}(H)=\gamma_{t}(H)$.

Proof. 1. We conclude from the assumptions that $\gamma_{d v e}(G) \geq \gamma_{d v e}(H)+s=\gamma_{t}(H)+s \geq \gamma_{t}(G)$ and this leads to the result in (1).

2. By inequality (1) and the assumptions, we have

$$
\gamma_{t}(G)=\gamma_{d v e}(G) \leq \gamma_{d v e}(H)+s \leq \gamma_{t}(H)+s \leq \gamma_{t}(G)
$$

Thus, all inequalities occurring in the above chain become equalities. In particular, $\gamma_{d v e}(H)=$ $\gamma_{t}(H)$.

The proof of next result is similar to the proof of Observation 3, and therefore we omit the details.

Observation 4. Let $H$ be a subgraph of a graph $G$.

1. If $\gamma_{2}(H)=\gamma_{d v e}(H), \gamma_{2}(G) \leq \gamma_{2}(H)+s$ and $\gamma_{d v e}(G) \geq \gamma_{d v e}(H)+$ s for some non-negative integer s, then $\gamma_{2}(G)=\gamma_{\text {dve }}(G)$.

2. If $\gamma_{2}(G)=\gamma_{d v e}(G), \gamma_{d v e}(G) \leq \gamma_{d v e}(H)+s$ and $\gamma_{2}(G) \geq \gamma_{2}(H)+$ s for some non-negative integer $s$, then $\gamma_{2}(H)=\gamma_{d v e}(H)$.

We close this section with the following simple observation.

Observation 5. If $T$ is a tree of order $n \geq 4$ with $2 \leq \operatorname{diam}(T) \leq 3$, then $\gamma_{t}(T)=\gamma_{d v e}(T)=2$ and $\gamma_{d v e}(T) \neq \gamma_{2}(T)$.

\section{Trees $T$ with $\gamma_{d v e}(T)=\gamma_{2}(T)$}

In this section, we provide a constructive characterization of all trees $T$ with $\gamma_{d v e}(T)=\gamma_{2}(T)$. For this purpose, we define the family $\mathcal{F}$ of unlabeled trees $T$ that can be obtained from a sequence $T_{1}, T_{2}, \ldots, T_{k}(k \geq 1)$ of trees such that $T_{1} \in\left\{P_{2}, P_{3}\right\}$ and $T=T_{k}$. If $k \geq 2, T_{i+1}$ can be obtained recursively from $T_{i}$ by one of the following operations.

Operation $\mathcal{T}_{1}$ : If $u \in V\left(T_{i}\right)$ and there is a pendant path $u x_{1} x_{2}$ such that $\operatorname{deg}_{T_{i}}\left(x_{1}\right)=2$ and $\operatorname{deg}_{T_{i}}\left(x_{2}\right)=$ 1 , then $\mathcal{T}_{1}$ adds a path $P_{2}=y_{1} y_{2}$ and an edge $u y_{1}$ to obtain $T_{i+1}$.

Operation $\mathcal{T}_{2}$ : If $u \in V\left(T_{i}\right)$ and there is a pendant path $u x_{1} x_{2}$ such that $\operatorname{deg}_{T_{i}}\left(x_{1}\right)=2$ and $\operatorname{deg}_{T_{i}}\left(x_{2}\right)=$ 1 , then $\mathcal{T}_{2}$ adds a path $P_{3}=y_{1} y_{2} y_{3}$ and an edge $u y_{1}$ to obtain $T_{i+1}$.

Operation $\mathcal{T}_{3}$ : If $u \in V\left(T_{i}\right)$, then $\mathcal{T}_{3}$ adds a copy of the graph $H_{k}$ for some $k \geq 2$, connected by an edge from its center vertex $a$ to $u$ to obtain $T_{i+1}$.

Operation $\mathcal{T}_{4}$ : If $u \in W_{T_{i}}^{1} \cap W_{T_{i}}^{4}$, then $\mathcal{T}_{4}$ adds a path $P_{5}=x_{1} x_{2} x_{3} x_{4} x_{5}$ and an edge $u x_{2}$ to obtain $T_{i+1}$. Operation $\mathcal{T}_{5}$ : If $u \in W_{T_{i}}^{3}$, then $\mathcal{T}_{5}$ adds a path $P_{4}=x_{1} x_{2} x_{3} x_{4}$ and an edge $u x_{1}$ to obtain $T_{i+1}$.

Operation $\mathcal{T}_{6}$ : If $u \in W_{T_{i}}^{4}$ and there is a pendant path $u x_{1} x_{2} x_{3}$, then $\mathcal{T}_{6}$ adds a path $P_{3}=y_{1} y y_{2}$ and an edge $u y$ to obtain $T_{i+1}$.

Lemma 1. If $T_{i}$ is a tree with $\gamma_{d v e}\left(T_{i}\right)=\gamma_{2}\left(T_{i}\right)$ and $T_{i+1}$ is a tree obtained from $T_{i}$ by one of the operations $\mathcal{T}_{i}(1 \leq i \leq 6)$, then $\gamma_{\text {dve }}\left(T_{i+1}\right)=\gamma_{2}\left(T_{i+1}\right)$. 
Proof. If $T_{i+1}$ is a tree obtained from $T_{i}$ by Operation $\mathcal{T}_{i}(1 \leq i \leq 3)$, then the result follows by Propositions 4, 6, and 8.

Let $T_{i+1}$ be a tree obtained from $T_{i}$ by Operation $\mathcal{T}_{4}$. Clearly, any $\gamma_{2}\left(T_{i}\right)$-set can be extended to a $2 \mathrm{~d}$-set of $T_{i+1}$ by adding $x_{1}, x_{3}, x_{5}$ and so $\gamma_{2}\left(T_{i+1}\right) \leq \gamma_{2}\left(T_{i}\right)+3$. Now, let $D$ be a $\gamma_{d v e}\left(T_{i+1}\right)$-set containing no leaves. Clearly, to dve-dominate the edge $x_{4} x_{5}$, we must have $x_{3}, x_{4} \in D$. If $D \cap$ $\left\{x_{1}, x_{2}\right\}=\varnothing$, then to dve-dominate the edge $x_{1} x_{2}$ we must have $u \in D$ and as $u \in W_{T_{i}}^{4}$, we have $\left|D \backslash\left\{x_{3}, x_{4}\right\}\right| \geq \gamma_{d v e}\left(T_{i}\right)+1$. If $D \cap\left\{x_{1}, x_{2}\right\} \neq \varnothing$, then $\left(D \backslash\left\{x_{1}, x_{2}, x_{3}, x_{4}\right\}\right) \cup\{u\}$ is an almost dve-dominating set of $T_{i}$ and, as above, we have $\left|\left(D \backslash\left\{x_{1}, x_{2}, x_{3}, x_{4}\right\}\right) \cup\{u\}\right| \geq \gamma_{d v e}\left(T_{i}\right)+1$. In either case, $\gamma_{d v e}\left(T_{i+1}\right) \geq \gamma_{d v e}\left(T_{i}\right)+3$. Therefore we conclude from Observation 4 (Item 1) that $\gamma_{2}\left(T_{i+1}\right)=\gamma_{d v e}\left(T_{i+1}\right)$.

Next, let $T_{i+1}$ be a tree obtained from $T_{i}$ by Operation $\mathcal{T}_{5}$. As $u \in W_{T_{i}}^{3}$, any $\gamma_{2}\left(T_{i}\right)$-set containing $u$ can be extended to a $2 \mathrm{~d}$-set of $T_{i+1}$ by adding $x_{2}, x_{4}$, and thus $\gamma_{2}\left(T_{i+1}\right) \leq \gamma_{2}\left(T_{i}\right)+2$. On the other hand, by Proposition 5, we have $\gamma_{d v e}\left(T_{i+1}\right) \geq \gamma_{d v e}\left(T_{i}\right)+2$. Therefore, by Observation 4 (Item 1), we obtain $\gamma_{2}\left(T_{i+1}\right)=\gamma_{d v e}\left(T_{i+1}\right)$.

Finally, let $T_{i+1}$ be a tree obtained from $T_{i}$ by Operation $\mathcal{T}_{6}$. Clearl,y any $\gamma_{2}\left(T_{i}\right)$-set can be extended to a $2 \mathrm{~d}$-set of $T_{i+1}$ by adding $y_{1}, y_{2}$, and so $\gamma_{2}\left(T_{i+1}\right) \leq \gamma_{2}\left(T_{i}\right)+2$. On the other hand, let $D$ be a $\gamma_{d v e}\left(T_{i+1}\right)$-set containing no leaves. Clearly, to dve-dominate the edges $y y_{1}, y y_{2}$ and $x_{2} x_{3}$, we must have $u, y, x_{1}, x_{2} \in D$. Then, $D-\{y\}$ is a dve-dominating set of $T^{\prime}$. As $u \in W_{T_{i}}^{4}$, we deduce that $|D-\{y\}| \geq \gamma_{d v e}\left(T_{i}\right)+1$. Therefore, $\gamma_{d v e}\left(T_{i+1}\right) \geq \gamma_{d v e}\left(T_{i}\right)+2$. By Observation 4 (Item 1), we obtain $\gamma_{2}\left(T_{i+1}\right)=\gamma_{d v e}\left(T_{i+1}\right)$.

Theorem 1. If $T \in \mathcal{F}$, then $\gamma_{2}(T)=\gamma_{\text {dve }}(T)$.

Proof. Let $T \in \mathcal{F}$. Then there exists a sequence of trees $T_{1}, T_{2}, \ldots, T_{k}(k \geq 1)$, such that $T_{1} \in\left\{P_{2}, P_{3}\right\}$ and $T=T_{k}$. Moreover, if $k \geq 2$, then $T_{i+1}$ can be obtained from $T_{i}$ by one of the aforementioned operations. We proceed by induction on the number of operations used to construct $T$. If $k=1$, then $T=T_{1} \in\left\{P_{2}, P_{3}\right\}$ and clearly $\gamma_{2}(T)=\gamma_{d v e}(T)$. Let $k \geq 2$, and assume that the result holds for each tree $T \in \mathcal{F}$, which can be obtained from a sequence of operations of length $k-1$. Let $T^{\prime}=T_{k-1}$. By the induction hypothesis, $\gamma_{2}\left(T^{\prime}\right)=\gamma_{d v e}\left(T^{\prime}\right)$. As $T=T_{k}$ is obtained by one of the Operations $\mathcal{T}_{i}$ $(i=1,2,3,4,5,6)$ from $T^{\prime}$, we conclude from Lemma 1 that $\gamma_{2}(T)=\gamma_{d v e}(T)$.

Before stating the main theorem of this section, we give the following two useful observations.

Observation 6. In a connected nontrivial graph $G$, every 2-dominating set of $G$ is a dve-dominating set.

Proof. Let $S$ be a 2-dominating set of $G$, and let $e=x y$ be any edge of $G$. Clearly, if $|S \cap\{u, v\}|=2$, then $e$ is ve-dominated once by $x$ and once by $y$. Therefore, we assume, without loss of generality, that $x \in S$. As $x$ has at least two neighbors in $S$, edge, $e$ is then ve-dominated at least twice by vertices in $N(x) \cap S$. In either case, $e$ is ve-dominated twice by vertices of $S$, and desired result follows.

Observation 7. Let $T$ be a tree of order $n \geq 4$ with $\gamma_{2}(T)=\gamma_{d v e}(T)$. Then, every support vertex of $T$ is adjacent to at most two leaves.

Proof. Let $D$ be a $\gamma_{2}(T)$-set, and let $v$ be a support vertex of $T$ such that $\left|L_{v}\right| \geq 3$. Clearly, $L_{v} \subseteq D$, and for every $w \in L_{v}, D-\{w\}$ is a dve-dominating set of $T$. Therefore, $\gamma_{d v e}(T) \leq|D|-1=\gamma_{2}(T)-1$, contradicting the fact $\gamma_{2}(T)=\gamma_{d v e}(T)$.

Theorem 2. Let $T$ be a tree of order $n \geq 2$. Then $\gamma_{2}(T)=\gamma_{\text {dve }}(T)$ if and only if $T \in \mathcal{F}$.

Proof. By Theorem 1, we only need to prove necessity. Let $T$ be a nontrivial tree of order $n$ with $\gamma_{2}(T)=\gamma_{d v e}(T)$. We proceed by induction on $n$. If $n \leq 3$, then $T \in\left\{P_{2}, P_{3}\right\}$ and $T \in \mathcal{F}$. Therefore, let $n \geq 4$. By Observation 5 , we have $\operatorname{diam}(T) \geq 4$, and thus $n \geq 5$. Assume that the result holds for 
every nontrivial tree $T^{\prime}$ having an order less than $n$ and satisfying $\gamma_{2}\left(T^{\prime}\right)=\gamma_{d v e}\left(T^{\prime}\right)$. Let $T$ be a tree of order $n$ with $\gamma_{2}(T)=\gamma_{d v e}(T)$. Let $v_{1} v_{2} \ldots v_{k}(k \geq 5)$ be a diametral path in $T$, such that $\operatorname{deg}_{T}\left(v_{2}\right)$ is as large as possible. Among such paths, we choose one so that $\operatorname{deg}_{T}\left(v_{3}\right)$ is as large as possible and root $T$ at $v_{k}$. By Observation $7,2 \leq \operatorname{deg}_{T}\left(v_{2}\right) \leq 3$. We consider the following cases.

Case 1. $\operatorname{deg}_{T}\left(v_{2}\right)=2$. By the choice of diametrical path, we may assume that any child of $v_{3}$ with depth one has degree 2 . We distinguish the following situations.

Subcase 1.1. $\operatorname{deg}_{T}\left(v_{3}\right) \geq 3$ and $v_{3}$ has a child $z$ with depth 0 .

Let $T^{\prime}=T-\{z\}$. Let $D$ be a $\gamma_{2}(T)$-set. Clearly $v_{1}, z \in D$ and $\left|D \cap\left\{v_{2}, v_{3}\right\}\right|=1$. Without loss of generality, we may assume that $v_{3} \in D$. Therefore, $D \backslash\{z\}$ is a $2 \mathrm{~d}$-set of $T^{\prime}$, which implies that $\gamma_{2}(T) \geq \gamma_{2}\left(T^{\prime}\right)+1$. On the other hand, any $\gamma_{d v e}\left(T^{\prime}\right)$-dominating set of $T^{\prime}$ containing no leaves is a dve-dominating set of $T$ implying that $\gamma_{d v e}(T) \leq \gamma_{d v e}\left(T^{\prime}\right)$. This leads to the contradiction $\gamma_{2}(T)-1 \geq \gamma_{2}\left(T^{\prime}\right) \geq \gamma_{d v e}\left(T^{\prime}\right) \geq \gamma_{d v e}(T)=\gamma_{2}(T)$.

Subcase 1.2. $\operatorname{deg}_{T}\left(v_{3}\right) \geq 3$ and $v_{3}$ has a child with depth 1 .

Let $T^{\prime}=T-T_{v_{2}}$. As there is a $\gamma_{2}(T)$-set $D$ containing $v_{3}, D \backslash\left\{v_{1}\right\}$ is a $2 \mathrm{~d}$-set of $T^{\prime}$ and so $\gamma_{2}(T) \geq$ $\gamma_{2}\left(T^{\prime}\right)+1$. On the other hand, if $S$ is a $\gamma_{d v e}\left(T^{\prime}\right)$-set containing no leaves, then $v_{3} \in S$ and thus $S \cup\left\{v_{2}\right\}$ is a dve-dominating set of $T$. Therefore, $\gamma_{d v e}(T) \leq \gamma_{d v e}\left(T^{\prime}\right)+1$. By Observation 4 (Item 2), we have $\gamma_{2}\left(T^{\prime}\right)=\gamma_{\text {dve }}\left(T^{\prime}\right)$. It follows from the induction hypothesis that $T^{\prime} \in \mathcal{F}$. Therefore, $T \in \mathcal{F}$, as it can be obtained from $T^{\prime}$ by Operation $\mathcal{T}_{1}$.

Subcase 1.3. $\operatorname{deg}_{T}\left(v_{3}\right)=2$ and $v_{4}$ has at least one child with depth 1 and degree 2 .

Let $T^{\prime}=T-T_{v_{3}}$. By Proposition 6 and by the assumption $\gamma_{2}(T)=\gamma_{d v e}(T)$, we have $\gamma_{2}\left(T^{\prime}\right)=\gamma_{d v e}\left(T^{\prime}\right)$. By the induction hypothesis, we have $T^{\prime} \in \mathcal{T}$, and as $T$ can be obtained from $T^{\prime}$ by Operation $\mathcal{T}_{2}$, we deduce that $T \in \mathcal{F}$.

Subcase 1.4. $\operatorname{deg}_{T}\left(v_{3}\right)=2$ and $v_{4}$ has a child $y$ with depth 1 and at least degree 3 .

By Observation $7, \operatorname{deg}_{T}(y)=3$. Let $L_{y}=\left\{y_{1}, y_{2}\right\}$ and $T^{\prime}=T-T_{y}$. Let $D$ be a $\gamma_{2}(T)$-set. Clearly, $y_{1}, y_{2} \in D$. If $y \in D$, then $\left(D \backslash\left\{y, y_{1}, y_{2}\right\}\right) \cup\left\{v_{4}\right\}$ is a $2 d$-set of $T^{\prime}$ and if $y \notin D$, then $D \backslash\left\{y_{1}, y_{2}\right\}$ is a 2d-set of $T^{\prime}$. In either case, we obtain $\gamma_{2}(T) \geq \gamma_{2}\left(T^{\prime}\right)+2$. Now, let $S$ be a $\gamma_{d v e}\left(T^{\prime}\right)$-set. If $v_{4} \in S$, then $S \cup\{y\}$ is a dve-dominating set of $T$, which leads to a contradiction as $\gamma_{2}(T) \geq \gamma_{2}\left(T^{\prime}\right)+2 \geq$ $\gamma_{d v e}\left(T^{\prime}\right)+2 \geq \gamma_{d v e}(T)-1+2=\gamma_{2}(T)+1$. Thus $v_{4} \notin S$ and so $v_{4} \in W_{T^{\prime}}^{4}$. On the other hand, as $S \cup\left\{v_{4}, y\right\}$ is a dve-dominating set of $T$, we have $\gamma_{d v e}(T) \leq \gamma_{d v e}\left(T^{\prime}\right)+2$. By Observation 4 (Item 2), we obtain $\gamma_{2}\left(T^{\prime}\right)=\gamma_{d v e}\left(T^{\prime}\right)$, and so by the induction hypothesis, we have $T^{\prime} \in \mathcal{F}$. Therefore, $T \in \mathcal{F}$ because it can be obtained from $T^{\prime}$ by Operation $\mathcal{T}_{6}$.

Subcase 1.5. $\operatorname{deg}_{T}\left(v_{3}\right)=2$ and $v_{4}$ has $\ell \geq 1$ children with depth 0 and $k \geq 2$ children with depth 2.

Let $T^{\prime}=T-T_{v_{4}}$. Assume that $v_{4} x_{1}^{i} x_{2}^{i} x_{3}^{i}(1 \leq i \leq k)$ are pendant paths in $T$. By the choice of diametrical path, we have $\operatorname{deg}\left(x_{1}^{i}\right)=\operatorname{deg}\left(x_{2}^{i}\right)=2$ for each $i \in\{1, \ldots, k\}$. We may assume that $\left\{x_{1}^{i}, x_{3}^{i} \mid 1 \leq i \leq k\right\} \cup L_{v_{4}}$ is a subset of any $\gamma_{2}(T)$-set. Let $D$ be a $\gamma_{2}(T)$-set. If $v_{4} \in D$, then replace $v_{4}$ by $v_{5}$ in $D$. Therefore, we can assume that $v_{4} \notin D$. It follows that $D \backslash\left(\left\{x_{1}^{i}, x_{3}^{i} \mid 1 \leq i \leq k\right\} \cup L_{v_{4}}\right)$ is a $2 d$-set of $T^{\prime}$ and thus $\gamma_{2}(T) \geq \gamma_{2}\left(T^{\prime}\right)+2 k+\ell$. On the other hand, any $\gamma_{d v e}\left(T^{\prime}\right)$-set can be extended to a dve-dominating set of $T$ by adding the vertices $x_{1}^{i}, x_{2}^{i}(1 \leq i \leq k)$, which implies that $\gamma_{d v e}(T) \leq \gamma_{d v e}\left(T^{\prime}\right)+2 k$. This leads to the following contradiction,

$$
\gamma_{2}(T)=\gamma_{d v e}(T) \leq \gamma_{d v e}\left(T^{\prime}\right)+2 k \leq \gamma_{2}\left(T^{\prime}\right)+2 k \leq \gamma_{2}(T)-\ell .
$$

Subcase 1.6. $\operatorname{deg}_{T}\left(v_{3}\right)=2$ and $v_{4}$ has $\ell \geq 2$ children with depth 0 and $k=1$ children with depth 2 .

Let $T^{\prime}=T-T_{v_{4}}$. As in the Subcase 1.5, we can see that $\gamma_{2}(T) \geq \gamma_{2}\left(T^{\prime}\right)+2+\ell$. Also, if $S$ is a $\gamma_{d v e}\left(T^{\prime}\right)$-set, then $S \cup\left\{v_{2}, v_{3}, v_{4}\right\}$ is a dve-dominating set of $T$, and thus $\gamma_{d v e}(T) \leq \gamma_{d v e}\left(T^{\prime}\right)+3$. Therefore,

$$
\gamma_{2}(T)=\gamma_{d v e}(T) \leq \gamma_{d v e}\left(T^{\prime}\right)+3 \leq \gamma_{2}\left(T^{\prime}\right)+3 \leq \gamma_{2}(T)-2-\ell+3,
$$


a contradiction.

Subcase 1.7. $\operatorname{deg}_{T}\left(v_{3}\right)=2$ and $v_{4}$ has no child with depth 0 and $k \geq 2$ children with depth 2 . Let $T^{\prime}=T-T_{v_{4}}$. Assume that $v_{4} x_{1}^{i} x_{2}^{i} x_{3}^{i}(1 \leq i \leq k)$ are pendant paths in $T$. By the choice of diametrical path, we have $\operatorname{deg}\left(x_{1}^{i}\right)=\operatorname{deg}\left(x_{2}^{i}\right)=2$ for each $i$. Thus, $T_{v_{4}}$ is isomorphic to the graph $H_{k}$. As in Subcase 1.5, we can see that $\gamma_{2}(T) \geq \gamma_{2}\left(T^{\prime}\right)+2 k$. On the other hand, any $\gamma_{d v e}\left(T^{\prime}\right)$-set can be extended to a dve-dominating set of $T$ by adding $x_{1}^{i}, x_{2}^{i}(1 \leq i \leq k)$ which implies that $\gamma_{d v e}(T) \leq \gamma_{d v e}\left(T^{\prime}\right)+2 k$. By Observation 4 (Item 2), we have $\gamma_{2}\left(T^{\prime}\right)=\gamma_{d v e}\left(T^{\prime}\right)$. It follows from the induction hypothesis that $T^{\prime} \in \mathcal{F}$. Therefore, $T \in \mathcal{F}$ because it can be obtained from $T^{\prime}$ by Operation $\mathcal{T}_{3}$.

Subcase 1.8. $\operatorname{deg}_{T}\left(v_{3}\right)=2$ and $v_{4}$ has exactly one child of depth 0 and one child of depth 2. Let $w$ be the leaf adjacent to $v_{4}$ and let $T^{\prime}=T-T_{v_{4}}$. Note that $T^{\prime}$ is nontrivial, for otherwise $\gamma_{2}(T) \neq \gamma_{d v e}(T)$. As in Subcase 1.5, we can see that $\gamma_{2}(T) \geq \gamma_{2}\left(T^{\prime}\right)+3$. Now, let $S$ be a $\gamma_{d v e}\left(T^{\prime}\right)$-set. If $v_{5} \in S$, then $S \cup\left\{v_{2}, v_{3}\right\}$ is a dve-dominating set of $T$ and we get the following contradiction,

$$
\gamma_{2}(T)=\gamma_{d v e}(T) \leq \gamma_{d v e}\left(T^{\prime}\right)+2 \leq \gamma_{2}\left(T^{\prime}\right)+2 \leq \gamma_{2}(T)-3+2
$$

Therefore, $v_{5} \notin S$, and thus $v_{5} \in W_{T^{\prime}}^{4}$. Then, $S \cup\left\{v_{2}, v_{3}, v_{4}\right\}$ is a dve-dominating set of $T$, implying that $\gamma_{\text {dve }}(T) \leq \gamma_{d v e}\left(T^{\prime}\right)+3$. By Observation 4 (item 2$)$, we have $\gamma_{2}\left(T^{\prime}\right)=\gamma_{d v e}\left(T^{\prime}\right)$, and thus $\gamma_{2}(T)=$ $\gamma_{2}\left(T^{\prime}\right)+3$ and $\gamma_{d v e}(T)=\gamma_{d v e}\left(T^{\prime}\right)+3$. We deduce from the induction hypothesis that $T^{\prime} \in \mathcal{T}$. Next, we need to prove that $v_{5} \in W_{T^{\prime}}^{1}$. Suppose, to the contrary, that $v_{5} \notin W_{T^{\prime}}^{1}$. Then, any $\gamma_{d v e}\left(T^{\prime} ; v_{5}\right)$-set can be extended to a dve-dominating set of $T$ by adding $v_{2}, v_{3}, v_{4}$, which leads to the contradiction

$$
\gamma_{d v e}(T) \leq \gamma_{d v e}\left(T^{\prime} ; v_{5}\right)+3 \leq \gamma_{d v e}\left(T^{\prime}\right)-1+3=\gamma_{d v e}\left(T^{\prime}\right)+2
$$

Therefore, $v_{5} \in W_{T^{\prime}}^{1}$, and thus $v_{5} \in W_{T^{\prime}}^{1} \cap W_{T^{\prime}}^{4}$. Consequently, $T$ can be obtained from $T^{\prime}$ by Operation $\mathcal{T}_{4}$, yielding $T \in \mathcal{F}$.

Subcase 1.9. $\operatorname{deg}_{T}\left(v_{3}\right)=2$ and $\operatorname{deg}_{T}\left(v_{4}\right)=2$. We distinguish the following situations.

(i) $\operatorname{deg}_{T}\left(v_{5}\right)=2$ or $v_{5}$ has a child with depth 0 or depth 1 or depth 2 .

Let $T^{\prime}=T-T_{v_{4}}$. As in Subcase 1.5, we have $\gamma_{2}(T) \geq \gamma_{2}\left(T^{\prime}\right)+2$. Now, let $S$ be a $\gamma_{d v e}\left(T^{\prime}\right)$-set containing no leaf. Clearly, $S \cap N_{T^{\prime}}\left[v_{5}\right] \neq \varnothing$, and thus $S \cup\left\{v_{2}, v_{3}\right\}$ is a dve-dominating set of $T$, which implies that $\gamma_{d v e}(T) \leq \gamma_{d v e}\left(T^{\prime}\right)+2$. By Observation 4 (Item 2) and the induction hypothesis, we obtain $T^{\prime} \in \mathcal{F}$. Next, we prove that $v_{5} \in W_{T^{\prime}}^{3}$. Suppose, to the contrary, that $v_{5} \notin W_{T^{\prime}}^{3}$, and let $D$ be a $\gamma_{2}(T)$-set. Without loss of generality, we may assume that $v_{1}, v_{3}, v_{5} \in D$. Then, $D \backslash\left\{v_{1}, v_{3}\right\}$ is a $2 \mathrm{~d}$-set of $T^{\prime}$ containing $v_{5}$, implying that $\gamma_{2}(T)-2=|D|-2 \geq \gamma_{2}\left(T^{\prime}\right)+1$. Therefore,

$$
\gamma_{d v e}(T)=\gamma_{2}(T) \geq \gamma_{2}\left(T^{\prime}\right)+3 \geq \gamma_{d v e}\left(T^{\prime}\right)+3 \geq \gamma_{d v e}(T)+1,
$$

a contradiction. Therefore, $v_{5} \in W_{T^{\prime}}^{3}$. Therefore, $T \in \mathcal{F}$ because it is obtained from $T^{\prime}$ by Operation $\mathcal{T}_{5}$.

(ii) $\operatorname{deg}_{T}\left(v_{5}\right) \geq 3$ and all children of $v_{5}$ are of depth 3 .

Let $v_{5} y_{4} y_{3} y_{2} y_{1}$ be a path in $T$ such that $y_{4} \notin\left\{v_{4}, v_{6}\right\}$. By seeing the previous cases, we have $\operatorname{deg}_{T}\left(y_{4}\right)=\operatorname{deg}_{T}\left(y_{3}\right)=\operatorname{deg}_{T}\left(y_{2}\right)=2$. Let $T^{\prime}=T-T_{v_{4}}$. As the Subcase 1.5, we have $\gamma_{2}(T) \geq$ $\gamma_{2}\left(T^{\prime}\right)+2$. Let $S$ be a $\gamma_{d v e}\left(T^{\prime}\right)$-set containing no leaf such that $\left|S \cap\left\{y_{2}, y_{3}, y_{4}\right\}\right|$ is as small as possible. Clearly $y_{2}, y_{3} \in D$ and by the choice of $S$, we have $v_{4} \notin S$. Therefore, $S \cup\left\{v_{2}, v_{3}\right\}$ is a dve-dominating set of $T$ and thus $\gamma_{d v e}(T) \leq \gamma_{d v e}\left(T^{\prime}\right)+2$. As in Item (i), we obtain $\gamma_{2}\left(T^{\prime}\right)=$ $\gamma_{d v e}\left(T^{\prime}\right), T^{\prime} \in \mathcal{F}$, and $v_{5} \in W_{T^{\prime}}^{3}$. Therefore, $T \in \mathcal{F}$, because it is obtained from $T^{\prime}$ by Operation $\mathcal{T}_{5}$.

Case 2. $\operatorname{deg}_{T}\left(v_{2}\right)=3$.

Assume that $L_{v_{2}}=\left\{v_{1}, w\right\}$. We consider the following subcases.

Subcase 2.1. $\operatorname{deg}\left(v_{3}\right) \geq 3$ and $v_{3}$ has a child with depth 1 , say $y$, with $y \neq v_{2}$.

Let $T^{\prime}=T-T_{v_{2}}$. Assume that $D$ is a $\gamma_{2}(T)$-set. If $v_{2} \in D$, then $D \backslash\left\{v_{1}, v_{2}, w\right\} \cup\left\{v_{3}\right\}$ is a $2 \mathrm{~d}$-set of 
$T^{\prime}$, and if $v_{2} \notin D$, then $D \backslash\left\{v_{1}, w\right\}$ is a $2 \mathrm{~d}$-set of $T^{\prime}$; so, in either case, $\gamma_{2}(T) \geq \gamma_{2}\left(T^{\prime}\right)+2$. Now, let $S$ be a $\gamma_{d v e}\left(T^{\prime}\right)$-set containing no leaves. As $y, v_{3} \in S, S \cup\left\{v_{2}\right\}$ is a dve-dominating set of $T$, and thus $\gamma_{\text {dve }}(T) \leq \gamma_{\text {dve }}\left(T^{\prime}\right)+1$., it follows that

$$
\gamma_{2}(T)=\gamma_{d v e}(T) \leq \gamma_{d v e}\left(T^{\prime}\right)+1 \leq \gamma_{2}\left(T^{\prime}\right)+1 \leq \gamma_{2}(T)-1,
$$

a contradiction.

Subcase 2.2. $\operatorname{deg}\left(v_{3}\right) \geq 3$ and $v_{3}$ has $\ell \geq 1$ children with depth 0 .

Let $D$ be a $\gamma_{2}(T)$-set. Then $\left\{v_{1}, w\right\} \cup L_{v_{3}} \subset D$, and thus $\left\{v_{2}, v_{3}\right\} \cup D-\left(\left\{v_{1}, w\right\} \cup L_{v_{3}}\right)$ is a dve-dominating set of $T$ of cardinality at most $\gamma_{2}(T)-1$, a contradiction.

Subcase 2.3. $\operatorname{deg}\left(v_{3}\right)=2$.

Let $D$ be a $\gamma_{2}(T)$-set $D$. Clearly, $D$ contains $v_{1}, w$. Moreover, to 2-dominate $v_{3}$, we must have $\left\{v_{2}, v_{3}\right\} \cap$ $D \neq \varnothing_{\text {, }}$ say $v_{3} \in D$. However, then, $\left\{v_{2}\right\} \cup D-\left\{v_{1}, w\right\}$ is a dve-dominating set of $T$ of cardinality at most $\gamma_{2}(T)-1$, a contradiction.

\section{Trees $T$ with $\gamma_{d v e}(T)=\gamma_{t}(T)$}

In this section, we provide a constructive characterization of all trees $T$ with $\gamma_{d v e}(T)=\gamma_{t}(T)$. For this purpose, we define the family $\mathcal{T}$ of unlabeled trees $T$ that can be obtained from a sequence $T_{1}, T_{2}, \ldots, T_{k}(k \geq 1)$ of trees, such that $T_{1} \in\left\{P_{2}, P_{3}, P_{4}\right\}$ and $T=T_{k}$. If $k \geq 2$, then $T_{i+1}$ can be obtained recursively from $T_{i}$ by one of the following operations.

Operation $\mathcal{O}_{1}$ : If $u \in V\left(T_{i}\right)$ is a support vertex or a non-leaf vertex adjacent to an end support vertex, then $\mathcal{O}_{1}$ adds a vertex $x$ and an edge $u x$ to obtain $T_{i+1}$.

Operation $\mathcal{O}_{2}$ : If $u \in V\left(T_{i}\right)$ and there is a path $u x_{1} x_{2}$ in $T_{i}$ such that $\operatorname{deg}_{T_{i}}\left(x_{1}\right)=2$ and $\operatorname{deg}_{T_{i}}\left(x_{2}\right)=1$, then $\mathcal{O}_{2}$ adds a path $P_{2}=y_{1} y_{2}$ and an edge $u y_{1}$ to obtain $T_{i+1}$.

Operation $\mathcal{O}_{3}$ : If $u \in W_{T_{i}}^{2}$, then $\mathcal{O}_{3}$ adds a path $P_{4}=x_{1} x_{2} x_{3} x_{4}$ and an edge $u x_{1}$ to obtain $T_{i+1}$.

Operation $\mathcal{O}_{4}$ : If $u \in V\left(T_{i}\right)$ and there is a path $u x_{1} x_{2}$ in $T_{i}$ such that $\operatorname{deg}_{T_{i}}\left(x_{1}\right)=2$ and $\operatorname{deg}_{T_{i}}\left(x_{2}\right)=1$, then $\mathcal{O}_{4}$ adds a path $P_{3}=y_{1} y_{2} y_{3}$ and an edge $u y_{1}$ to obtain $T_{i+1}$.

Operation $\mathcal{O}_{5}$ : If $u \in W_{T_{i}}^{1}$ and there is a path $u x_{1} x_{2} x_{3}$ in $T_{i}$ such that $\operatorname{deg}_{T_{i}}\left(x_{1}\right)=\operatorname{deg}_{T_{i}}\left(x_{2}\right)=2$ and $\operatorname{deg}_{T_{i}}\left(x_{3}\right)=1$, then $\mathcal{O}_{5}$ adds a path $y_{1} y_{2} y_{3}$ and an edge $u y_{1}$ to obtain $T_{i+1}$.

Operation $\mathcal{O}_{6}$ : If $u \in V\left(T_{i}\right)$ is a leaf and there is a path $u x_{1} x_{2} x_{3}$ in $T_{i}$ such that $\operatorname{deg}_{T_{i}}\left(x_{3}\right)=1$ and no $\gamma_{\text {dve }}\left(T_{i}\right)$-set contains both $u$ and $x_{1}$, then $\mathcal{O}_{6}$ adds a vertex $x$ and an edge $u x$ to obtain $T_{i+1}$.

Operation $\mathcal{O}_{7}$ : If $u \in V\left(T_{i}\right)$ is a support vertex with $v \in L_{u}$ and there is a path $u y_{2} y_{1}$ such that $\operatorname{deg}_{T_{i}}\left(y_{2}\right)=2$ and $\operatorname{deg}_{T_{i}}\left(y_{1}\right)=1$, then $\mathcal{O}_{7}$ adds a new vertex $w$ and a path $P_{4}=x_{1} x_{2} x_{3} x_{4}$ and edges $y_{1} w, v x_{4}$ to obtain $T_{i+1}$.

Lemma 2. If $T_{i}$ is a tree with $\gamma_{\text {dve }}\left(T_{i}\right)=\gamma_{t}\left(T_{i}\right)$ and $T_{i+1}$ is a tree obtained from $T_{i}$ by one of the operations $\mathcal{O}_{j}(1 \leq j \leq 7)$, then $\gamma_{d v e}\left(T_{i+1}\right)=\gamma_{t}\left(T_{i+1}\right)$.

Proof. If $T_{i+1}$ is a tree obtained from $T_{i}$ by Operation $\mathcal{O}_{j}$, where $j \in\{1, \ldots, 5\}$, then the result follows by Propositions 3, 4, 5, 6, and 7.

Let $T_{i+1}$ be obtained from $T_{i}$ by Operation $\mathcal{O}_{6}$. Clearly, any $\gamma_{t}\left(T_{i}\right)$-set can be extended to a td-set of $T$ by adding $u$ which implies that $\gamma_{t}\left(T_{i+1}\right) \leq \gamma_{t}\left(T_{i}\right)+1$. Moreover, if $D$ is a $\gamma_{d v e}\left(T_{i+1}\right)$-set containing no leaf, then clearly $u, x_{1} \in D$ and $D$ is a double ve-dominating set of $T_{i}$. As, by the assumption in Operation $\mathcal{O}_{6}$, no $\gamma_{d v e}\left(T_{i}\right)$-set contains both $u$ and $x_{1}$, we deduce that $|D|>\gamma_{d v e}\left(T_{i}\right)_{\text {, }}$ that is, $\gamma_{d v e}\left(T_{i+1}\right) \geq \gamma_{d v e}\left(T_{i}\right)+1$. Now, by Observation 3 (Item 1), we obtain $\gamma_{d v e}\left(T_{i+1}\right)=\gamma_{t}\left(T_{i+1}\right)$.

Assume now that $T_{i+1}$ is obtained from $T_{i}$ by Operation $\mathcal{O}_{7}$. As any $\gamma_{t}\left(T_{i}\right)$-set can be extended to a td-set of $T$ by adding $x_{2}, x_{3}, y_{1}$, we have $\gamma_{t}\left(T_{i+1}\right) \leq \gamma_{t}\left(T_{i}\right)+3$. Let $D$ be a $\gamma_{d v e}\left(T_{i+1}\right)$-set containing no leaf. Then, $y_{1}, y_{2}, x_{2}, x_{3} \in D$, and to dve-dominate the edge $v x_{4}$, we may assume that $u \in D$. 
Therefore, $D-\left\{y_{1}, x_{2}, x_{3}\right\}$ is a dve-dominating set of $T_{i}$, implying that $\gamma_{d v e}\left(T_{i+1}\right) \geq \gamma_{d v e}\left(T_{i}\right)+3$. By Observation 3 (Item 1), we obtain $\gamma_{d v e}\left(T_{i+1}\right)=\gamma_{t}\left(T_{i+1}\right)$.

Now we are ready to state the main theorem of this section.

Theorem 3. Let $T$ be a tree of order $n \geq 2$. Then $\gamma_{\text {dve }}(T)=\gamma_{t}(T)$ if and only if $T \in \mathcal{T}$.

Proof. Let $T \in \mathcal{T}$. Then there exists a sequence of trees $T_{1}, T_{2}, \ldots, T_{k}(k \geq 1)$, such that $T_{1} \in\left\{P_{2}, P_{3}, P_{4}\right\}$ and $T=T_{k}$. Moreover, if $k \geq 2$, then $T_{i+1}$ can be obtained from $T_{i}$ by one of the aforementioned operations. We proceed by induction on the number of operations used to construct $T$. If $k=1$, then $T \in\left\{P_{2}, P_{3}, P_{4}\right\}$ and clearly $\gamma_{d v e}(T)=\gamma_{t}(T)$. Assume now that $k \geq 2$ and that the result holds for each tree, $T \in \mathcal{T}$, that can be obtained from a sequence of length $k-1$, and let $T^{\prime}=T_{k-1}$. By the induction hypothesis, $\gamma_{\text {dve }}\left(T^{\prime}\right)=\gamma_{t}\left(T^{\prime}\right)$. As $T=T_{k}$ is obtained from $T^{\prime}$ by using one of the Operations $\mathcal{O}_{j}$, where $j \in\{1,2, \ldots, 7\}$, we conclude from the Lemma 2 that $\gamma_{d v e}(T)=\gamma_{t}(T)$.

Conversely, let $\gamma_{d v e}(T)=\gamma_{t}(T)$. We proceed by induction on $n$. If $n \leq 3$, then $T \in\left\{P_{2}, P_{3}\right\}$ and clearly $T \in \mathcal{T}$. Let $n \geq 4$ and let the result hold for every tree $T^{\prime}$ of order less than $n$, satisfying $\gamma_{\text {dve }}\left(T^{\prime}\right)=\gamma_{t}\left(T^{\prime}\right)$. Let $T$ be a tree of order $n$ with $\gamma_{d v e}(T)=\gamma_{t}(T)$. If $\operatorname{diam}(T)=2$, then $T$ is a star, and it can be obtained from $P_{3}$ by frequently use of Operation $\mathcal{O}_{1}$, and so $T \in \mathcal{T}$. If $\operatorname{diam}(T)=3$, then $T$ is a double star $D S_{p, q},(q \geq p \geq 1)$. If $q=1$, then $T=P_{4} \in \mathcal{T}$. If $q>1$, then $T$ can be obtained from $P_{4}$ by frequently use of $\mathcal{O}_{1}$, and thus $T \in \mathcal{T}$. Henceforth, we assume that $\operatorname{diam}(T) \geq 4$. Let $v_{1} v_{2} \ldots v_{k}(k \geq 5)$ be a diametral path in $T$ such that $\operatorname{deg}_{T}\left(v_{2}\right)$ is as large as possible. Root $T$ at $v_{k}$.

If $\operatorname{deg}_{T}\left(v_{2}\right) \geq 3$, then let $T^{\prime}=T-\left\{v_{1}\right\}$. By Proposition 3 and by the induction hypothesis, we have $T^{\prime} \in \mathcal{T}$. Now, $T$ can be obtained from $T^{\prime}$ by operation $\mathcal{O}_{1}$ and so $T \in \mathcal{T}$. Therefore, we assume that $\operatorname{deg}_{T}\left(v_{2}\right)=2$. By the choice of diametrical path, we may assume that any child of $v_{3}$ with depth one is of degree two. Note that using an argument similar to the previous one, we may assume that $T$ has no strong support vertex. We consider the following cases.

Case 1. $\operatorname{deg}_{T}\left(v_{3}\right) \geq 3$.

If $v_{3}$ has at least one child with depth 0 , say $x$, then let $T^{\prime}=T-\{x\}$. By Proposition 3 and by the induction hypothesis, we have $T^{\prime} \in \mathcal{T}$. It follows that $T \in \mathcal{T}$ because it is obtained from $T^{\prime}$ by operation $\mathcal{O}_{1}$. Therefore, we assume that $v_{3}$ is not a support vertex. Then, $v_{3}$ has at least one children with depth 1 , say $z_{2}$, different from $v_{2}$. Let $v_{3} z_{2} z_{1}$ be a pendant path in $T$. Suppose $T^{\prime}=T-\left\{v_{1}, v_{2}\right\}$. By Proposition 4 and by the induction hypothesis, we have $T^{\prime} \in \mathcal{T}$. Now, as $T$ is obtained from $T^{\prime}$ by

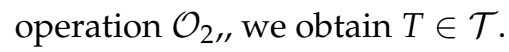

Case 2. $\operatorname{deg}_{T}\left(v_{3}\right)=2$.

First, let $\operatorname{deg}_{T}\left(v_{4}\right) \geq 3$. If $v_{4}$ has a child $y_{2}$ with depth 1 , then let $v_{4} y_{2} y_{1}$ be a pendant path in $T$, and let $T^{\prime}=T-T_{v_{3}}$. By Proposition 6 and the inductive hypothesis on $T^{\prime}$, we have $T^{\prime} \in \mathcal{T}$. It follows that $T \in \mathcal{T}$, as it can be obtained from $T^{\prime}$ by operation $\mathcal{O}_{4}$.

Assume now that $v_{4}$ has a child $z_{3}$ with depth 2, different from $v_{3}$. According to the previous case, the path $v_{4} z_{3} z_{2} z_{1}$, having $z_{1}$ as a leaf, is a pendant path in $T$. Let $T^{\prime}=T-T_{v_{3}}$. By Proposition 7 and Observation 3 (item 2), we have $\gamma_{d v e}\left(T^{\prime}\right)=\gamma_{t}\left(T^{\prime}\right), \gamma_{t}(T)=\gamma_{t}\left(T^{\prime}\right)+2$ and $\gamma_{d v e}(T)=\gamma_{d v e}\left(T^{\prime}\right)+2$. By the induction hypothesis on $T^{\prime}$, we have $T^{\prime} \in \mathcal{T}$. We prove now that $v_{4} \in W_{T^{\prime}}^{1}$. Suppose, to the contrary, that $v_{4} \notin W_{T^{\prime}}^{1}$. Then $\gamma_{d v e}\left(T^{\prime} ; v_{4}\right) \leq \gamma_{d v e}\left(T^{\prime}\right)-1$ and any $\gamma_{d v e}\left(T^{\prime} ; v_{4}\right)$-set can be extended to a dve-dominating set of $T$ by adding $v_{2}, v_{3}$ implying that $\gamma_{d v e}(T) \leq \gamma_{d v e}\left(T^{\prime} ; v_{4}\right)+2 \leq \gamma_{d v e}\left(T^{\prime}\right)-1+2=$ $\gamma_{d v e}\left(T^{\prime}\right)+1$, a contradiction. Therefore, $v_{4} \in W_{T^{\prime}}^{1}$, and therefore $T \in \mathcal{T}$ because it can be obtained from $T^{\prime}$ by operation $\mathcal{O}_{5}$.

Finally, suppose $v_{4}$ is a support vertex, and let $T^{\prime}=T-\left\{v_{1}\right\}$. Clearly, for any $\gamma_{t}(T)$-set $D$ containing no leaf, we have $v_{2}, v_{3}, v_{4} \in D$, and thus $D \backslash\left\{v_{2}\right\}$ is a td-set of $T^{\prime}$, implying that $\gamma_{t}(T) \geq$ $\gamma_{t}\left(T^{\prime}\right)+1$. On the other hand, any $\gamma_{\text {dve }}\left(T^{\prime}\right)$-set containing no leaves, contains $v_{3}, v_{4}$ and thus such a set plus $v_{2}$ is a dve-dominating set of $T$, implying that $\gamma_{d v e}(T) \leq \gamma_{d v e}\left(T^{\prime}\right)+1$. By Observation 3 (Item 2 ), we have $\gamma_{\text {dve }}\left(T^{\prime}\right)=\gamma_{t}\left(T^{\prime}\right)$. By the induction hypothesis, we have $T^{\prime} \in \mathcal{T}$. Note that Observation 3 (Item 2) also implies that $\gamma_{d v e}(T)=\gamma_{d v e}\left(T^{\prime}\right)+1$. This means that no $\gamma_{d v e}\left(T^{\prime}\right)$-set $S$ contains both $v_{2}$ 
and $v_{3}$; otherwise, $S$ dve-dominates $T$ too, which contradicts $\gamma_{d v e}(T)=\gamma_{d v e}\left(T^{\prime}\right)+1$. Now, as $T$ can be obtained from $T^{\prime}$ by operation $\mathcal{O}_{6}$, we conclude that $T \in \mathcal{T}$.

From now on, we can assume that $\operatorname{deg}_{T}\left(v_{4}\right)=2$. We distinguish the following subcases.

Subcase 2.1. $\operatorname{deg}_{T}\left(v_{5}\right) \geq 3$.

Let $T^{\prime}=T-T_{v_{4}}$. By Proposition 5, we have $\gamma_{t}(T)=\gamma_{t}\left(T^{\prime}\right)+2$. Let $S$ be a $\gamma_{d v e}\left(T^{\prime}\right)$-set containing no leaves. If $v_{5}$ is a support vertex or is adjacent to a support vertex, then clearly $\left|S \cap N_{T^{\prime}}\left[v_{5}\right]\right| \geq 1$. If there is a path $v_{5} z_{3} z_{2} z_{1}$ such that $z_{3} \notin\left\{v_{4}, v_{6}\right\}$ and $\operatorname{deg}\left(z_{1}\right)=1$, then $z_{3} \in S$, and thus $\left|S \cap N_{T^{\prime}}\left[v_{5}\right]\right| \geq 1$. If there is a path $v_{5} z_{4} z_{3} z_{2} z_{1}$ such that $z_{4} \notin\left\{v_{4}, v_{6}\right\}$ and $\operatorname{deg}\left(z_{1}\right)=1$, then, as $z_{1}$ belongs to diametral path of $T$, each of $z_{4}, z_{3}$ and $z_{2}$ has degree two, and thus $z_{2}, z_{3} \in S$. However, to ve-dominate the edge $v_{5} z_{4}$ we need that $\left|S \cap N_{T^{\prime}}\left[v_{5}\right]\right| \geq 1$. In either case, we deduce that $v_{5} \in W_{T^{\prime}}^{2}$. In this case, $S \cup\left\{v_{2}, v_{3}\right\}$ is a dve-dominating set of $T$, implying that $\gamma_{d v e}(T) \leq \gamma_{d v e}\left(T^{\prime}\right)+2$. By Observation 3 (Item 2), we obtain $\gamma_{t}\left(T^{\prime}\right)=\gamma_{d v e}\left(T^{\prime}\right)$, and it follows from the induction hypothesis that $T^{\prime} \in \mathcal{T}$. Therefore, $T \in \mathcal{T}$ because it is obtained from $T^{\prime}$ by Operation $\mathcal{O}_{3}$.

Subcase 2.1. $\operatorname{deg}_{T}\left(v_{5}\right)=2$.

We note that if $v_{6}$ is a leaf, then $T=P_{6}$ that belongs to $\mathcal{T}$, as it can be obtained from a path $P_{2}$ (whose vertices are in $W_{P_{2}}^{2}$ ) by using Operation $\mathcal{O}_{3}$. Therefore, we assume that $\operatorname{deg}_{T}\left(v_{6}\right) \geq 2$. We consider some further situations.

(a) $v_{6}$ has a child with depth 0 or 1 or $\operatorname{deg}_{T}\left(v_{6}\right)=2$.

Let $T^{\prime}=T-T_{v_{4}}$. By Proposition 5, $\gamma_{t}(T)=\gamma_{t}\left(T^{\prime}\right)+2$ and $\gamma_{d v e}(T) \geq \gamma_{d v e}\left(T^{\prime}\right)+2$. On the other hand, any $\gamma_{d v e}\left(T^{\prime}\right)$-set containing no leaves can be extended to a dve-dominating set of $T$ by adding two vertices, $v_{2}$ and $v_{3}$, which implies that $\gamma_{d v e}(T) \leq \gamma_{d v e}\left(T^{\prime}\right)+2$. By Observation 3 (Item 2), we obtain $\gamma_{t}\left(T^{\prime}\right)=\gamma_{d v e}\left(T^{\prime}\right)$, and it follows from the induction hypothesis that $T^{\prime} \in \mathcal{T}$. Also, by assumption, it is clear that $v_{5} \in W_{T^{\prime}}^{2}$, and therefore $T \in \mathcal{T}$, as it can be obtained from $T^{\prime}$ by Operation $\mathrm{O}_{3}$.

(b) $v_{6}$ has a child with depth 3.

Let $y_{4}$ be a child of $v_{6}$ with depth 3 such that $v_{6} y_{4} y_{3} y_{2} y_{1}$ is a path in $T$. As $T$ has no strong support vertex, we have $\operatorname{deg}\left(y_{2}\right)=2$. If $\operatorname{deg}\left(y_{3}\right) \geq 3$, then by a similar argument to that used in Case 1 , we can easily see that $T \in \mathcal{T}$. Therefore, we may assume that $\operatorname{deg}\left(y_{3}\right)=2$. If $\operatorname{deg}\left(y_{4}\right) \geq 3$, then, again, by using a similar argument as in the beginning of Case 2, we have $T \in \mathcal{T}$. Thus, we may assume that $\operatorname{deg}\left(y_{4}\right)=2$. Now, let $T^{\prime}=T-T_{y_{4}}$. By Proposition 5, we have $\gamma_{t}(T)=\gamma_{t}\left(T^{\prime}\right)+2$. Let $S$ be a $\gamma_{d v e}\left(T^{\prime}\right)$-set containing no leaves. Then, $v_{2}, v_{3} \in S$, and to dve-dominate the edge $v_{4} v_{5}$, we must have $S \cap\left\{v_{4}, v_{5}, v_{6}\right\} \neq \varnothing$, say $v_{6} \in S$. Hence $v_{6} \in W_{T^{\prime}}^{2}$. Also, $S \cup\left\{y_{2}, y_{3}\right\}$ is a dve-dominating set of $T$, and thus $\gamma_{d v e}(T) \leq \gamma_{d v e}\left(T^{\prime}\right)+2$. By Observation 3 (Item 2), we obtain $\gamma_{t}\left(T^{\prime}\right)=\gamma_{d v e}\left(T^{\prime}\right)$, , and by the induction hypothesis, we obtain $T^{\prime} \in \mathcal{T}$. Therefore, $T \in \mathcal{T}$, as it is obtained from $T^{\prime}$ by Operation $\mathcal{O}_{3}$.

(c) $v_{6}$ has a child with depth 4, different from $v_{5}$.

Let $y_{5}$ be a child of $v_{6}$ with depth 4 such that $v_{6} y_{5} y_{4} y_{3} y_{2} y_{1}$ is a path in $T$. Note that, as $y_{1}$ belongs to a diametral path of $T$, and seeing the above situations, we can assume that $v_{6} y_{5} y_{4} y_{3} y_{2} y_{1}$ is a pendant path. Let $T^{\prime}=T-T_{y_{4}}$. By Proposition 5, $\gamma_{t}(T)=\gamma_{t}\left(T^{\prime}\right)+2$. Let $S$ be a $\gamma_{\text {dve }}\left(T^{\prime}\right)$-set containing no leaves. As above, $v_{2}, v_{3} \in S$, and we may assume that $v_{6} \in S$ to double ve-dominating the edge $v_{4} v_{5}$. Thus $y_{5} \in W_{T^{\prime}}^{2}$ and $S \cup\left\{y_{2}, y_{3}\right\}$ is a dve-dominating set of $T$,, implying that $\gamma_{d v e}(T) \leq \gamma_{d v e}\left(T^{\prime}\right)+2$. By Observation 3 (Item 2), we obtain $\gamma_{t}\left(T^{\prime}\right)=\gamma_{d v e}\left(T^{\prime}\right)$, and thus $T^{\prime} \in \mathcal{T}$. Now, as $T$ is obtained from $T^{\prime}$ by Operation $\mathcal{O}_{3}$, we deduce that $T \in \mathcal{T}$.

(d) $v_{6}$ has a child with depth 2 .

Considering above situations, we may assume that any child of $v_{6}$ other than $v_{5}$ has depth 2 . First, let $\operatorname{deg}_{T}\left(v_{6}\right)=3$. Let $y_{3}$ be the child of $v_{6}$ with depth 2 such that $v_{6} y_{3} y_{2} y_{1}$ is a path in $T$. As $T$ has no strong support vertex we have $\operatorname{deg}_{T}\left(y_{2}\right)=2$. Also, if $\operatorname{deg}_{T}\left(y_{3}\right) \geq 3$, then, as in the Case 1 , we can see that $T \in \mathcal{T}$. Therefore, we may assume that $\operatorname{deg}_{T}\left(y_{3}\right)=2$. Let $T^{\prime}$ be the tree obtained from $T$ by removing the set of vertices $\left\{y_{1}, v_{1}, v_{2}, v_{3}, v_{4}\right\}$. If $D$ is a $\gamma_{t}(T)$-set containing no leaf, 
then $y_{3}, y_{2}, v_{2}, v_{3} \in D$ and to dominate $v_{5}$, we may assume that $v_{6} \in D$. Thus, $D-\left\{v_{2}, v_{3}, y_{1}\right\}$ is a td-set of $T^{\prime}$, and thus $\gamma_{t}(T) \geq \gamma_{t}\left(T^{\prime}\right)+3$. On the other hand, any $\gamma_{d v e}\left(T^{\prime}\right)$-set containing no leaf contains $y_{3}, v_{6}$, and thus can be extended to a dve-dominating set of $T$ by adding $v_{2}, v_{3}, y_{2}$. Therefore, $\gamma_{d v e}(T) \leq \gamma_{d v e}\left(T^{\prime}\right)+3$. By Observation $3\left(\right.$ Item 2), we have $\gamma_{t}\left(T^{\prime}\right)=\gamma_{d v e}\left(T^{\prime}\right)$, and thus by our inductive hypothesis, $T^{\prime} \in \mathcal{T}$. As $T$ can be obtained from $T^{\prime}$, by using Operation $\mathcal{O}_{7}$, we deduce that $T \in \mathcal{T}$.

Author Contributions: F.M. and S.M.S. contributed to the supervision, validation, project administration and formal analysis. W.F, M.C. and R.K. contributed to the methodology and investigation and wrote the initial draft of the paper, which was investigated and approved by M.C., M.S., and the final draft was written by S.M.S. and M.S.

Funding: This work is supported by the Scientific Research Fund of SiChuan Provincial Department of Science and Technology (2019YFS0067), the Open Project Program of the State Key Lab of CAD \& CG under Grant A1922 (Zhejiang University), and the Scientific Research Fund of Key Laboratory of Pattern Recognition and Intelligent Information Processing of Chengdu University(MSSB-2019-03).

Conflicts of Interest: The authors declare no conflict of interest.

\section{References}

1. Haynes, T.W.; Hedetniemi, S.T.; Slater, P.J. Fundamentals of Domination in Graphs; Marcel Dekker, Inc.: New York, NY, USA, 1998.

2. Henning, M.A.; Yeo, A. Total Domination in Graphs (Springer Monographs in Mathematics); Springer: Berlin, Germany, 2013. ISBN (Print) 978-1-4614-6524-9, ISBN (Online) 978-1-4614-6525-6.

3. Peters, J.W. Theoretical and Algorithmic Results on Domination and Connectivity. Ph.D. Thesis, Clemson University, Clemson, SC, USA, 1986.

4. Ahangar, H.A.; Chellali, M.; Sheikholeslami, S.M.; Soroudi, M.; Volkmann, L. Total vertex-edge domination in trees. Ars Combin 2019, submitted.

5. Boutrig, R.; Chellali, M.; Haynes, T.W.; Hedetniemi, S.T. Vertex-edge domination in graphs. Aequat. Math. 2016, 90, 355-366. [CrossRef]

6. Chellali, M.; Haynes, T.W.; Hedetniemi, S.T.; Lewis, T.M. On ve-degrees and ev-degrees in graphs. Discrete Math. 2017, 340, 31-38. [CrossRef]

7. Krishnakumari, B.; Venkatakrishnan, Y.B.; Krzywkowski, M. Bounds on the vertex-edge domination number of a tree. C. R. Acad. Sci. Paris, Ser. I 2014, 352, 363-366. [CrossRef]

8. Lewis, J.R. Vertex-Edge and Edge-Vertex Domination in Graphs. Ph.D. Thesis, Clemson University, Clemson, SC, USA, 2007.

9. Lewis, J.R.; Hedetniemi, S.T.; Haynes, T.W.; Fricke, G.H. Vertex-edge domination. Util. Math. 2010, 81, 193-213.

10. Zyliński, P. Vertex-edge domination in graphs. Aequat. Math. 2019, 93, 735-742. [CrossRef]

11. Krishnakumari, B.; Chellali, M.; Venkatakrishnan, Y.B.; Double vertex-edge domination. Discrete Math. Algorithms Appl. 2017, 9, 1750045. [CrossRef]

(c) 2019 by the authors. Licensee MDPI, Basel, Switzerland. This article is an open access article distributed under the terms and conditions of the Creative Commons Attribution (CC BY) license (http:/ / creativecommons.org/licenses/by/4.0/). 